\title{
SUPERNOVA OBSERVING WEB (SNOW) PROJECT WITH THE PUBLIC ASTRONOMICAL OBSERVATORIES IN JAPAN
}

\author{
HITOSHI YAMAOKA \\ Department of Physics, Faculty of Science, Kyushu University \\ 4-2-1 Ropponmatsu, Chuo-ku, Fukuoka 810, Japan \\ MAMORU DOI, TOSHIKAZU SHIGEYAMA, NAOKI YASUDA \\ Department of Astronomy, School of Science, University of \\ Tokyo, 2-11-6, Yayoi, Bunkyo-ku, Tokyo 113, Japan \\ AND \\ MASARU WATANABE \\ Astronomical Data Analysis Center, National Astronomical \\ Observatory Japan, 2-21-1, Osawa, Mitaka, Tokyo 181, Japan
}

We are promoting a supernova(SN) detecting project (named SNOW) with medium size $(60 \mathrm{~cm}-105 \mathrm{~cm})$ telescopes belonging to the public astronomical observatories in Japan. Main observations were started on January 1997 with the cooperation of seven observatories, and will be continued some years with more participants. One of our purposes is to determine the $\mathrm{SN}$ rate especially in early type galaxies, which plays an important role in the chemical and dynamical evolution of them and surrounding hot gases. Each telescope, with the $R$-band filter and chilled CCD camera, observes several fields allotted respectively for it. Fields are selected to include the dense cores of nearby $(z<0.1)$ clusters of galaxies, then each field contaiins about $10-20$ regular $\mathrm{E} / \mathrm{S} 0$ galaxies. In order not to miss the $\mathrm{SNe}$ emerging in the fields, every fields are checked within two weeks after the latest observation. The flatfielding and the detection of new (or brighter) objects are automated. To inform the monitoring status for compensating each other for lack of observation, a WWW page is organized (http://www.netwave.or.jp/ yfujita/snow.html, written in Japanese). It is also used for the publicity of the project. Unfortunately, we have not detected any new SNe in our field till October 1997. We expect that we will be able to determine the SN rate within the $35 \%$ error after two years spell, which will be reduced with a longer run or more entrants. 